\title{
The problem of morphogenesis: unscripted biophysical control systems in plants
}

\author{
Philip M. Lintilhac
}

Received: 11 June 2013 / Accepted: 14 June 2013 /Published online: 12 July 2013

(C) The Author(s) 2013. This article is published with open access at Springerlink.com

\begin{abstract}
The relative simplicity of plant developmental systems, having evolved within the universal constraints imposed by the plant cell wall, may allow us to outline a consistent developmental narrative that is not currently possible in the animal kingdom. In this article, I discuss three aspects of the development of the mature form in plants, approaching them in terms of the role played by the biophysics and mechanics of the cell wall during growth. First, I discuss axis extension in terms of a loss of stability-based model of cell wall stress relaxation and I introduce the possibility that cell wall stress relaxation can be modeled as a binary switch. Second, I consider meristem shape and surface conformation as a controlling element in the morphogenetic circuitry of plant organogenesis at the apex. Third, I approach the issue of reproductive differentiation and propose that the multicellular sporangium, a universal feature of land plants, acts as a stressmechanical lens, focusing growth-induced stresses to create a geometrically precise mechanical singularity that can serve as an inducing developmental signal triggering the initiation of reproductive differentiation. Lastly, I offer these three examples of biophysically integrated control processes as entry points into a narrative that provides an independent, nongenetic context for understanding the evolution of the apoplast and the morphogenetic ontogeny of multicellular land plants.
\end{abstract}

Keywords Morphogenesis $\cdot$ Loss of stability $\cdot$ Axis extension $\cdot$ Meristem shape $\cdot$ Surface conformation . Reproductive differentiation

Handling Editor: David Robinson

P. M. Lintilhac $(\bowtie)$

Department of Plant Biology, The University of Vermont,

63 Carrigan Drive, Burlington, VT 05405, USA

e-mail: Philip.Lintilhac@uvm.edu

\section{Introduction}

More than half a century after the discovery of the molecular basis for biological inheritance, we are still faced with the problem of understanding some of the most basic transformations that make up the developmental ontogeny of organisms. In plant biology, the answers to the most mundane and obvious questions underlying plant growth and morphogenesis still elude us. How do plant cells grow? What controls organogenesis on the apical meristem? How is reproductive differentiation initiated in plants? In many respects, our ability to understand living organisms at the molecular level may have outstripped our ability to place organismal development in the context of a reasonable and unifying system of functional controls.

The great German philosopher-poet Goethe, who coined the term morphogenesis, may have been the first to express the emergence of form in plants as an ongoing and continuous progression of changing shapes that point to some system of hidden rules governing the development of the complex form from preexisting embryonic structures (Mueller 1952). Understanding the nature of these rules has been one of the primary goals of botanical research. But there may be another reason for attempting to establish a general understanding of organismal development. In order to evaluate the significance of any new observation, whether molecular, cellular, or organismal, we have to be able to see how it fits into an overall developmental narrative, which we, therefore, need to articulate clearly. To quote Scheiner (2010):

"Science progresses faster when researchers operate within an explicit framework of concepts and theories."

Erwin Schrödinger may have been the first to seriously consider the thermodynamic constraints within which life evolved. In his seminal book "What is life?," he raised fundamental questions about organismal evolution and development (Schrödinger 1944). Subsequently, working within the framework of nonequilibrium thermodynamic systems, Katchalsky 
and Curran (1965) and others attempted more formal descriptions of living organisms couched in terms of hierarchically embedded feedback systems and subsystems (Eigen and Schuster 1979). More recent work has shown that these subsystems and their control pathways were probably inherited as more or less intact and functioning modules from prokaryotic pro-symbionts and subsequently tailored to meet the needs of the first true eukaryotic cells, which eventually fully integrated them by replicating, co-opting, or eliminating parts of their genomes.

True eukaryotic multicellularity appears to have arisen later (Grosberg and Strathmann 2007). But with the emergence of multicellularity, natural selection was subsequently able to modify individual pathways only to the extent that those changes altered the form and behavior of the whole organism as it progressed from one developmental stage to the next because as Takhtajan (1991)noted:

"Any evolutionary change in adult structure of organisms is the result of hereditary alterations in ontogeny in successive generations."

In other words, developmental ontogeny is the feedstock for evolution. The implication of this is clear. If we want to understand the evolution of plant form in any meaningful detail, then we will have to develop a firm understanding of developmental ontogeny-how plant form is produced moment to moment as the plant matures.

Furthermore, our ability to translate subsystem function into an understanding of whole organism development is fundamentally incomplete because, although each subsystem is maintained by its own internal control circuitry, none appears to be appropriately positioned to orchestrate development at the level of whole organism morphogenesis. This proposition, which has been called the Subsystem Incompleteness Theorem (Niklas and Kutschera 2012), highlights our inability to translate our understanding of events at the molecular and subcellular levels into a convincing narrative of organismal development.

In plants, however, the relative simplicity of development as compared to animals may afford a unique opportunity to bridge this gap. Several factors may contribute to our ability to read the logic of plant development in ways that are not possible in animals. First of all, the permanent recording of cell division behavior in tissue pattern allows us to reconstruct and interpret morphogenetic history directly; and secondly, as I hope to illustrate, development itself appears to be unified under a remarkable structural paradigm that is made possible by the universal presence of the apoplast.

\section{Plants vs. animals}

The basic eukaryotic cell plan probably originated in a unicellular lineage that was later adopted by both plants and animals (Grosberg and Strathmann 2007). We see evidence of this common origin in the extensive homologies between plants and animals at the molecular level. But plants are structural beings, and although genomics and biochemistry are fundamental to understanding their biology, it is becoming increasingly apparent that multicellular land plants evolved very differently from animals. Animal evolution seems to have organized itself around movement. Animals move in order to find food and shelter, and they need to actively interpret their surroundings in order to avoid predation. At the developmental level too, animal cell mitosis involves the pinching in half of the parent cell. The two "daughter" cells can then separate from each other, which frees them to migrate independently from one location to another. Cells slide past each other to create new neighborhoods, encounter new environments, and foster functional relationships in ways that are unavailable to plant cells (Walbot 1985).

When plants emerged onto dry land almost a billion years ago (Magallon et al. 2013), they encountered evolutionary pressures very different from those that channeled animal evolution. Competition for sunlight, soil nutrients, and water drove the evolution of the stem - the extension of a vertical axis between earth and sky. Plants do not need to move because photosynthesis excuses them from the need to locate and capture a meal. Plants have no need for an active fightor-flight response, and because of their sessile, autotrophic life histories, they do not require the sensory complexity and elaborate hormonal control systems found in animals. The need for rapid long-distance signaling is virtually nonexistent. There is no active imaging of the environment, no moving edge detection, no need to link explosive muscle contraction to visual or other inputs, no hunger, no fear, and none of the complex developmental controls underlying the interpretation of environment and social interaction.

\section{Constraints on plant cell behavior}

Construction of the typical plant body can be considered to be an architectural process. An analogy can be drawn between building a plant and building a skyscraper where new living space is created at the top, just as new cells are added in meristems located at the tips of branches. Plant cell division is very much like installing a new partition wall across an existing room. Walls must be accurately positioned and correctly oriented in order to support the loads. The construction materials must be matched to the loading conditions and the expected performance requirements.

Like adjacent rooms sharing a common wall, the individual cells making up the plant body are permanently bonded together and cannot move relative to each other. This system of structural wall materials (the "apoplast") extends throughout the plant, providing a strong mechanical coupling that binds all 
somatic cells together as a continuous structural entity. The fact that each cell is trapped in its own walled enclosure also makes it possible for plant cells to develop significant internal hydrostatic pressures. Turgor pressures in living plant cells are extreme by animal standards. Growing plant cells commonly maintain pressures in excess of 4 bar $(0.4 \mathrm{MPa})$, and some plant cells may have pressures in excess of $10 \mathrm{bar}(1.0 \mathrm{MPa})$ (Tomos and Leigh 1999). While these adaptations would appear to limit the repertoire of cellular behaviors that would be required for the kinds of developmental complexity we find in the animal kingdom, for plants, they represent the very features that have shaped multicellularity, features that are at the heart of developmental ontogeny in land plants. If we look for the probable origins of multicellularity in plants, we find that, notwithstanding the extensive commonalities at the subsystem level, the weight of evidence points to the fact that multicellularity evolved completely independently in plants and in animals (Grosberg and Strathmann 2007; Graham 1993).

Because of these profound divergences between the plant and animal kingdoms, it seems entirely reasonable to propose that the evolution and development of multicellular land plants has been driven by a structural logic that is very different from the movement-based logic of the animal kingdom (Walbot 1985). One might also conclude that, because of the relative functional simplicity of plant development, it may be possible to outline a developmental narrative in a way that cannot yet be achieved in animals - a narrative based on the physics and mechanics of the cell wall system.

\section{A brief history of the study of plant development}

The study of plant development has been hindered by the lack of a clear path of inference from the molecular to the organismal levels of organization. The physical sciences enjoy some degree of theoretical continuity between the symmetries of matter and energy at the subatomic level and our growing understanding of the organization of the cosmos as a whole, with the entire body of physical theory being held together by a consistent mathematical language whose strength lies in the fact that it is derived from first principles; but in the biological sciences, there is no such continuity. This is most clearly evident in the controversy that still lingers in the question of the role of the cell in plant development: Do the cells act as individual decision-making units, with the flow of development reflecting the consensus of the population, as if every cell were a citizen in the body politic of the organism, or does the control of development reside in the whole organism with cells simply being required to fill the space? Even now we puzzle over this question that was first debated by Matthias Schleiden and Anton DeBary over a hundred years ago (Kaplan and Hagemann 1991; Wojtaszek 2001).
Plant biology also has its own historical inertia to overcome. The traditions of morphology, anatomy, physiology, and genetics have imposed their own distinct perspectives on problems of growth, structural integrity, and sexual differentiation. Each of these disciplines originates in different methodological approaches. Each maintains its own vocabulary and terminology, traces its history through its own literature, claims its own heroes, and sees itself as the key to understanding development as a whole. More recently, the molecular biology of gene expression has given us a tool of great precision and power that can be applied to both animals and plants, a tool that can be used to dissect cellular controls and signaling pathways in ways that were previously unimaginable. But like the three blind men feeling different parts of the same elephant, we have to ask if there is something we have missed. Is it possible to elaborate a plant-specific paradigm that can bring all these subtexts together in a narrative that encompasses mechanism, form, evolution, and development?

\section{Towards a unifying logic of plant development}

First impressions are sometimes the most insightful. The most striking aspect of any section through living plant material is the prominent structural patterning at the tissue level and the implication that hidden in the pattern is some kind of developmental logic. The lack of cell mobility in plants also provides a unique window into developmental history because the patterns that we see in section constitute a frozen record of cell division activity. ${ }^{1}$

The first students of plant morphogenesis and organogenesis, working either through direct observation or with histological preparations, could see that many aspects of plant development strongly suggest that physical forces play a prominent role in development. They saw these forces playing out in the patterns of cells in sectioned material and they attempted to codify them in simple rules ${ }^{2}$ that could

\footnotetext{
${ }^{1}$ Early plant biologists were clearly impressed with the geometric and architectural nature of plant structures seen in section. Wilhelm Hofmeister, a largely self-taught microscopist and botanist, concluded that it was the epidermis enclosing the plant body that controlled and directed morphogenesis and organogenesis in plants (Kaplan and Cooke 1996). Sinnott (1960), summarizing early work on cell shape and patterning in plants, interpreted the geometrical cell wall arrangements that we see in early embryos and many other plant structures in terms of geometrical relationships and physical forces akin to surface tension forces.

${ }^{2}$ Hofmeister's rule: Hofmeister (1863) stated that, in general, the new partition wall is installed at right angles to the long axis of the cell. Sachs' rule: Julius von Sachs (1878) observed that the new partition wall meets the side walls at an angle of $90^{\circ}$, even if this means that the new wall must be a curved meniscus surface. Errera's rule: Leo Errera (1888), following Plateau's (1873) observation that bubble films tended to find configurations that minimize the surface area, proposed that partition walls divide the parent cell into equal volumes while adding the least new surface area.
} 
help them explain how cell behavior generated organic form. Many others have attempted to extract developmental meaning from the pattern information that is so clearly evident in plant materials. Hejnowicz $(1984,1993)$ and SzymanowskaPulka (2012) have developed models that tracked principal growth trajectories in roots, relating them to the patterns seen in sectioned material and implying that there is a direct link between growth directionality and organogenesis. The common theme that links all of these observations, both the old and the new, is that the governing principles of plant organogenesis and morphogenesis are more directly accessible through the study of pattern and the immediate causes of pattern generation, than they are through the detailing of molecular dynamics and subcellular control circuitry.

At the molecular level, all organisms tend to look quite similar. Antibodies to sea urchin sperm axonemes will recognize and bind with plant cell tubulin - an ancient handshake that predates multicellularity itself. One could, therefore, assume that the same developmental principles are at work in plants and in animals, with positional morphogens and signaling cascades operating in similar ways to coordinate the development of spatial order at the tissue level. But if multicellularity arose independently in plants, then we have to admit the possibility that plant development evolved its own unique set of regulatory tools and feedbacks governing the elaboration of multicellular form.

\section{The role of the cell wall in the coordination of plant development}

The ability to synthesize cellulose probably has a prokaryotic origin where it can still be found in bacteria including Acetobacter xylinum as well as a variety of eukaryotic unicells and marine algae. It can even be found in the animal kingdom in tunicates such as Ciona intestinalis (Nakashima et al. 2004). It was probably incorporated into early eukaryotic unicells as an endosymbiotic carryover, and for that reason, it was probably not the cellulosic wall itself that enabled the evolution of the land plant growth habit, but rather it was the ability to manipulate wall behavior that was the key (Niklas 2004). As Niklas suggests, it may have been the ability to fine-tune the behavior of the wall through the incorporation of specific proteins and other complex polysaccharides that endowed the streptophytes with the ability to control wall performance at the nanostructural level.

Primary wall extension growth, commonly referred to as "diffuse growth," is fundamental to plant morphogenesis and the evolution of shape. Indeed, the ability of plant cells to undergo rapid axial extension is the basis of what may be the single most important evolutionary invention of land plants, which is the ability to raise a vertical axis above the land surface. It is generally agreed that this is due to the individual cell's ability to constrain enlargement by controlling the orientation of the cellulose microfibrils of the cell wall. It is also agreed that, for cell volume increase to become fixed as permanent growth, it must be accompanied by some kind of stress relaxation because, otherwise, the enlarged cells would tend to recoil to their original size. The nature of this stress relaxation has been the subject of some debate however.

Attempts to work with isolated cell wall materials have found that, when uniaxial mechanical extension is imposed on excised wall material, the resulting wall stress decays exponentially over time. This time dependency of stress relaxation has been interpreted to indicate a viscoelastic stress relaxation response (Dorrington 1980; Taiz 1984; Metraux and Taiz 1978). The problem with this mechanism is that, although cell wall materials do indeed show the timedependent relaxation characteristic of viscoelasticity, it has only been observed under experimental conditions where a rapid loading is applied, resulting in a sudden extension. During normal plant cell growth, there is no sudden extension that can serve to initiate an ongoing viscoelastic wall relaxation. The tensile forces that drive wall extension in normal growing cells are generated by gradual, osmotically driven water potential changes, leaving plenty of time for stresses to equilibrate continuously.

In an attempt to develop a more universal model of stress relaxation behavior, we took a different approach to the problem (Wei and Lintilhac 2003). We looked for a way to understand stress relaxation in terms of general physical principles. We went back to the original work of Leonhard Euler (1707-1783), the great Swiss mathematician who in 1757 derived the mathematical basis for structural instability in columns under compression. Euler identified the necessary conditions for this instability in a constitutive equation that he derived from first principles, establishing the fact that even a flawless cylindrical column will become unstable and collapse when the load reaches a certain critical value. ${ }^{3}$ Euler's celebrated derivation makes it clear that the critical value depends solely upon the geometric and material properties of the column, and not upon the loading rate. As the load increases gradually, the column compresses longitudinally until at some point the smallest increase in load will result in runaway instability and the column will collapse in any one of a number of modes depending on the material. There are four key points here. First, the expression defining the critical force value at which instability occurs amounts to a prediction of the ultimate sustainable load. Second, in order for instability to be observed in the absence of some other form of catastrophic failure such as impact fracture, the

\footnotetext{
${ }^{3}$ For a slender column the critical force $F_{\mathrm{CR}}$ at which instability occurs is: $F_{\mathrm{CR}}=\frac{\Pi^{2} E I}{L^{2}}$, where $I$ is the area moment of inertia, $E$ is the elastic modulus, and $L$ is the length of the column.
} 
stressing load must be applied gradually. Third, although the load is applied gradually, the resulting instability is instantaneous and generally catastrophic ${ }^{4}$. Lastly, we should note that Euler's expression defining the parameters of instability was derived from first principles and, consequently, is applicable to any column under compression, regardless of its composition or microstructure, as long as it is loaded gradually.

In the twentieth century, the mathematical instability theory was extended to other structural configurations, first by Rzhanitsyn (1955), who applied it to columns in tension, and later by Panovko and Gubanova (1965), who published a detailed monograph applying the principles of instability theory to spherical pressure vessels ${ }^{5}$. Wei and Lintilhac (2007) reviewed this work in detail and derived an expression showing that the mathematics of instability can also be applied to a cylindrical pressure vessel, with obvious implications for the behavior of cylindrical plant cells and other plant structures whose elongation is driven by internal (turgor) pressure ${ }^{6}$.

The analogy with plant cells is inescapable. Growing plant cells are essentially pressure vessels whose behavior must be subject to the same physical laws as any pressure vessel. But why then does instability in a pressurized plant cell not lead to some form of irreversible failure as it would in a column under compression? Panovko addressed this issue directly. He pointed out that, in the case of a closed pressure vessel, any relaxation of the vessel wall would result in an incremental increase in volume, which would immediately lower the internal pressure and prevent runaway instability. In other words, because plant cells enclose an incompressible volume (water being essentially incompressible), even the smallest increase in volume will immediately

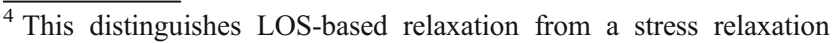
mechanism based on viscoelastic behavior, where the deforming load is applied rapidly and the resulting stress decays logarithmically over an extended period of time (Dorrington 1980).

${ }^{5}$ For a spherical pressure vessel, the critical pressure $P_{\mathrm{CR}}$ at which instability occurs is: $P_{\mathrm{CR}}=\frac{4 E T_{0}}{3 e R_{0}}$, where $E$ is the elastic modulus, $T_{0}$ is the initial thickness of the vessel wall, $R_{0}$ is the initial radius of the vessel, and $e$ is the natural constant 2.72 .

${ }^{6}$ For a cylindrical pressure vessel, the critical pressure value at which instability occurs is: $P_{\mathrm{CR}}=\frac{E T_{0}}{e R_{0}(1+v)}$, where $E$ and $v$ are the elastic modulus and Poisson's ratio of the wall, respectively, $t_{0}$ and $R_{0}$ are parameters determined by the wall thickness $t$ and cell radius $R$ for a given value of Poisson's ratio. This says that, for a given cylindrical pressure vessel with initial radius $R_{0}$, initial wall thickness $t_{0}$, and with elastic modulus $E$ and Poisson's ratio $v$, there is an internal pressure $P_{\mathrm{CR}}$ at which the cylinder wall will become unstable, precipitating instability-driven stress relaxation. Note that there is no term denoting time dependency in this expression. As long as the pressure is able to rise to the $P_{\mathrm{CR}}$ value, no matter how gradually, some form of instability will occur. Only if some other mechanism intervenes to prevent the internal pressure from attaining $P_{\mathrm{CR}}$ will instability be prevented. Note also that this expression says nothing about the detailed structure of the wall or how the instability will be manifested.
}

drop the internal pressure down to some value below $P_{\mathrm{CR}}$. This means that the mathematically derived model for instability in an enclosed pressure vessel is particularly relevant to our understanding of cell wall behavior in turgid plant cells. In growing plant cells, as turgor pressures and the associated wall stresses rise, they will eventually reach a point where some region of the wall becomes unstable and a local stress relaxation event occurs. Whatever the nature of the instability, the cell volume will increase slightly, dropping the internal pressure and the resulting wall stresses back down to some lower value and rescuing the cell from potential rupture (Fig. 1).

But now we are faced with another question. If wall relaxation proceeds by a cycle of increasing stress and sudden volume increase, why then does cell growth not appear to be a step function, with turgor rising smoothly to the value of $P_{\mathrm{CR}}$ followed by an instability-driven relaxation event and a sudden increase in volume? Common experience tells us that cell growth is a smooth process, whereas an instabilitydriven stress relaxation would seem to imply an intermittent growth. Again, Panovko shows us that, in the real world, no pressure vessel wall will be of perfectly uniform thickness over its entire surface, nor will its material properties be identical from one location to another. This means that instability-driven stress relaxation will never affect the entire cell at once, but will always be restricted to the locations where the value of $P_{\mathrm{CR}}$ is at its lowest. In any living plant cell, the natural texture of a composite wall will result in local variations in wall thickness and modulus, ensuring that instability-driven relaxation will not affect the entire cell wall at once.

This presents us with an entirely new way of thinking about the yield behavior of the wall in cells that are actively

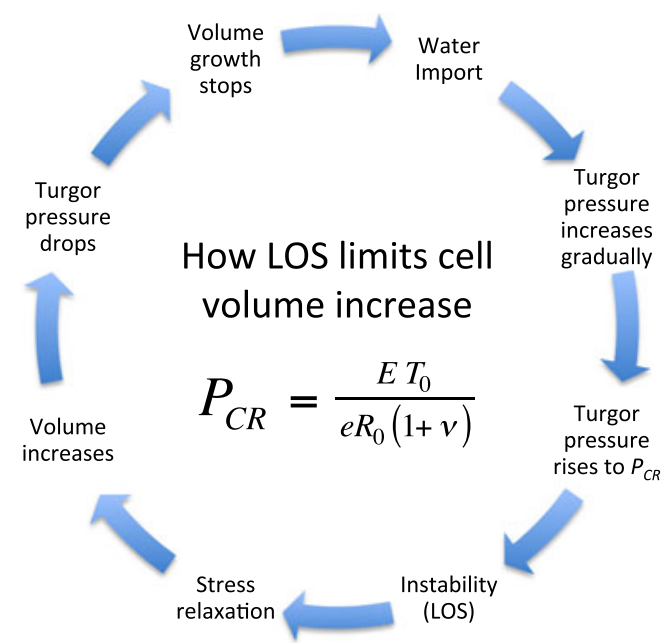

Fig. 1 LOS-driven stress relaxation in a cylindrical cell is limited by the increase in volume and the resulting pressure drop. The turgor pressure $P_{\mathrm{CR}}$ at which stress relaxation will occur is a function of the geometry and material properties of the cell wall 
growing. Rather than regarding stress relaxation as a viscoelastic process that is distributed more or less uniformly over the entire cell surface, with some areas simply being more or less stretchy than others, we now can imagine growth as being a patchy process, with the patches reflecting the inherent texture of the wall material, and instability-driven yielding events always occurring first in areas where the wall thickness or local modulus is the lowest. What may appear to us as the smooth "diffuse growth" we normally associate with extending walls in axially elongating tissues may be more accurately represented as "pixelated" growth, which is flickering over the texture of the cell wall, always picking out the locations where the critical pressure $\left(P_{\mathrm{CR}}\right)$ value is at its lowest. ${ }^{7}$ Thus, the pixel size defining local instability events may only have meaning at the nanoscale.

The most immediate inference that one can draw from this model of cell wall stress relaxation is that the critical pressure value determined by the mathematics of loss of stability (LOS) constitutes a precise turgor pressure prediction for growing cells because turgor can never rise above the value of $P_{\mathrm{CR}}$ without releasing stress relaxation and volume increase. The details of this derivation and the experimental confirmation of its predictive strength have been published elsewhere (Wei and Lintilhac 2007). We concluded that the LOS model is able to predict the turgor pressure of an actively growing cylindrical cell and, therefore, that it defines what we might call the working turgor pressure for growth. No other model of cell wall behavior has been able to do this.

Perhaps, more intriguing than the predictive potential of the LOS model is the possibility of re-imagining the control circuitry governing plant cell growth in a more general sense. We can now contemplate a process of cell and organ extension growth that is intrinsically self-limiting and which can be switched on and off locally by fine-tuning the composition of the wall and controlling the value of $P_{\mathrm{CR}}$. One can imagine local synthetic sites tracking across the inner wall surface, leaving a trail of slightly increased $P_{\mathrm{CR}}$ values. By slightly raising the value of $P_{\mathrm{CR}}$ locally, against a surrounding landscape of lower-valued pixels, the cell could switch off growth, forcing stress relaxation and wall extension to migrate to other regions of the cell and thereby controlling the deposition of wall thickenings in patterned cells (Hepler 1971).

\footnotetext{
${ }^{7}$ Instability can take many forms, and it may be initiated be any kind of general force, a distributed force (pressure) or a torque (moment). Panovko and Gubanova (1965) published a detailed monograph applying the principles of instability theory to other configurations. An example of a completely reversible instability is the bending of a carpenter's steel tape, where the U-shaped cross-section of the tape in the extended form is the stable equilibrium configuration until the force reaches a certain value at which point the original structure will collapse into a different equilibrium configuration. The curvature in the transverse section will disappear almost completely, but it can be restored when the tape is straightened.
}

To summarize:

1. The LOS model places plant cell growth behavior within reach of physical first principle, making it potentially applicable to any cell regardless of wall composition or geometry.

2. It implies that, for any local wall region for which the thickness and material properties can be determined, there is a precisely defined stress level stress at which instabilitydriven stress relaxation must occur. In a growing plant cell, the source of this tensile stress is turgor pressure.

3. Instability implies that stress relaxation is an on-or-off condition because, as long as the turgor pressure of a given cell is even slightly below the lowest $P_{\mathrm{CR}}$ value, stress relaxation cannot occur.

4. Instability-driven stress relaxation enables us to define the working turgor pressure as the pressure at which stress relaxation must occur.

5. It establishes the fact that the turgor pressure in any plant cell cannot rise above the value of $P_{\mathrm{CR}}$, implying that stress relaxation in plant cell walls should be treated as a binary switch and not as a threshold-based process.

6. The cell's ability to control wall biochemistry and texture takes on new meaning as a complex network of tuning elements that provides the cell with the means to manipulate the value of $P_{\mathrm{CR}}$ at the nanostructural and ultrastructural levels.

\section{The origin of shape}

There is a long history of invoking mechanical and structural signals as inputs into the progression of shapes that are generated as the apical meristem cycles through its sequence of forms (Selker et al. 1992; Lyndon 1994). We know that plant cells respond to mechanical signals in specific and predictable ways; first, by installing new cell partition walls in predictable orientations with respect to the mechanical forces acting on them (Lintilhac 1974) and, second, by controlling the anisotropy of wall synthesis and the directionality of cell enlargement. It seems clear that all of these factors likely play some role in determining the placement of young leaf primordia on the apical meristem. Hamant et al. (2008) and others have drawn attention to the correspondence between cytoskeletal dynamics, wall microfibril orientations, and organogenetic behavior. But what still remains undefined is the connection between these direct inputs into meristem behavior and the control pathways that are capable of maintaining the integrity of the system as a whole, providing for the continuity between successive manifestations of apical form.

It is in this context that I want to examine one aspect of apical morphogenesis that is rarely discussed and has never 
been approached systematically; that is, the role that shape itself plays in determining cell and tissue behavior. Meristematic shape has been considered to be the incidental end product of apical morphogenesis; simply the outward manifestation of processes that are going on at other levels of integration and, therefore, not a part of whatever control circuitry is responsible for the continuity of form in the apex. What I propose here is that shape, meaning the surface conformation of growing plant structures, is in itself an element in a feedback control circuit that is at the heart of morphogenesis in all land plants, and that, when we put it in its place among the other known factors that contribute to shape change, it completes a picture of meristematic behavior that is easily comprehended, self-sustaining, and perhaps unique to plants.

So, having established in the previous section that one of the most elemental features of land plant growth, namely, the ability to extend a vertical axis, can be approached in terms of a relatively simple LOS-based control cycle, let us now consider the second quintessential feature of land plant morphogenesis which is the functional behavior of the apical meristem itself. The most significant activity of the apical meristem is its ability to cycle through a repeated sequence of forms that, in the case of the shoot apex, consists of a regular pattern of leaf and axillary bud primordia originating as local changes in the conformation of the apical surface. Meristems are constantly changing shape. They are heavily invested in the generation of new surface. New cells contribute their division wall orientations to the maintenance of cell patterning and their enlarging volumes to the volume of the apex, while at the same time generating new surface that accelerates out of the apex and away from the apical pole. The continuous reshaping of the meristem is, thus, partly a function of new cell formation within the meristem and partly a function of the controlled cell enlargement. The contributions of each of these two aspects of growth are variable from one location to another. But while it is clear that cell division and enlargement act to generate shape, there is a lack of understanding of the role that shape itself plays. We need to consider the shape of the apex, meaning its surface topology, not just as the product of apical meristem behavior but also as a controlling element in a conformational feedback circuit. We need to consider shape as input as well as output.

When considering how the forces produced by enlarging cells are transmitted from cell to cell through a growing plant structure, we need to be aware of some very basic rules governing force transmission through a solid. We begin with the proposition that turgor-driven cell enlargement releases forces that propagate directionally through adjacent cellular tissues, and because the cells cannot move, the transmitted forces and their counterforces set up an equilibrium that can be mapped as a stress field comprised of mutually perpendicular "principal stresses."

- Transmitted force is stress.

- It is invisible, but it results in predictable combinations of tensile, compressive, or shear stresses being set up in the material.

- At any point in the material, these stresses can be resolved into mutually perpendicular tensile or compressive "principal stresses" representing the trajectories of pure compression or pure tension.

- The network of principal stresses that radiates through the structure forms a three-dimensional tensor field that defines the trajectories of pure tension and pure compression everywhere in the structure.

- The surface conformation (shape) of a structure determines the spatial configuration of the principal stresses just under the surface. For example:

- At any free boundary (surface), these stresses resolve into two families, those acting in the plane of the surface (i.e., parallel to the surface) and those acting perpendicular to the surface.

- When the boundary is convex to the outside (bulging out), the stresses acting in the plane of the surface will be of opposite sign to the normal stresses; for instance, if the stress in the surface plane is compressive, then the normal stresses will be tensile.

- When the boundary is concave, the normal and parallel stresses will be of the same sign (Heywood 1969).

This means that the bounding surface of a particular shape will determine the directions and, to some extent, the intensity of the principal stresses immediately beneath the surface. This is why the surface conformations of critically stressed engineering structures need to be carefully controlled to guide the flow of stresses and eliminate local stress risers.

Now, we need to consider the two components of growth that contribute to the generation of surface shape in plant meristems, namely, cell division and cell volume growth (Nick 2000). First, local mitoses make their own individual contributions to surface shape. Second, postmitotic cell enlargement results in the surface of the meristem being further deformed in predictable ways. These two inputs into surface shape appear to be strongly linked, and they probably share a common dependence on the cell cytoskeleton (Nick 2011; Hamant et al. 2008) for accurate mechanical transduction and for spatial information.

The precision with which plant cells and tissues control the orientation of new partition walls has been demonstrated. It can be seen in a variety of instances including the rotation of the cell plate during the division of cambial initials, but it 
is perhaps most clearly evident in the wound response (Lintilhac and Vesecky 1981). It has long been understood that the azimuthal controls of division wall installation are responsive to the mechanical environment of the dividing cell (Brown 1964). Furthermore, under conditions where the forces transmitted through an actively dividing tissue can be reliably resolved into principal stresses, the plane of the new partition wall is always normal to one of those principal stresses. ${ }^{8}$ The precision of this process has been confirmed under conditions where directional mechanical loads are applied to whole plants in vivo (Lintilhac and Vesecky 1981), to callus cultures in vitro (Lintilhac 1984), and even to isolated protoplasts (Lynch and Lintilhac 1997). In general, we know that, when the pattern of stresses acting through an actively dividing tissue is altered or disturbed, the orientations of the next generation of partition walls will conform to the new stress pattern.

Now, we can put these facts together in a deterministic control cycle. We know that the trajectories of principal stresses propagating near the meristem surface conform to surface topology, so that the shape of the meristem surface imposes its own imprint on the pattern of stresses acting in the subsurface layers. We also know that those same stress directionalities can act as azimuthal cues for the next round of cell divisions and cell enlargement. So, we can conclude that the surface conformation of the meristem must, therefore, be determining the orientation of the new partition wall installations that will, after the next round of divisions, lead to the reshaping of the meristem surface. This means that shape, principal stress patterning, and the two components of growth (volume growth and cell partitioning) are inextricably linked in a deterministic cycle that can function as a selfsustaining feedback circuit, maintaining a tight control over the progression of shape changes that is the fundamental developmental activity of the meristem (Fig. 2).

Because plant meristems are so small, they are inevitably highly invested in surface conformation. Everything is close to a surface. Also, because their cells are pressurized and tightly coupled and, therefore, transmitting the forces generated by their volume growth directly to their neighbors, they can use the spatial precision and predictability of mechanical inputs to control tissue behavior at the meristematic level. Mechanical stress can serve as the structural analog of a positional morphogen, but with a specificity and precision in space and time that is unavailable to systems based on diffusible molecules.

Although this interpretation of morphogenetic control in the meristem may seem antithetical to a more physiological and molecular interpretation of plant development, it may

\footnotetext{
${ }^{8}$ Lintilhac (1974) interpreted this behavior in terms of a simple operational rule whereby the dividing cell installs its new partition wall in the orientation which would subject it to the minimum shear stress.
}

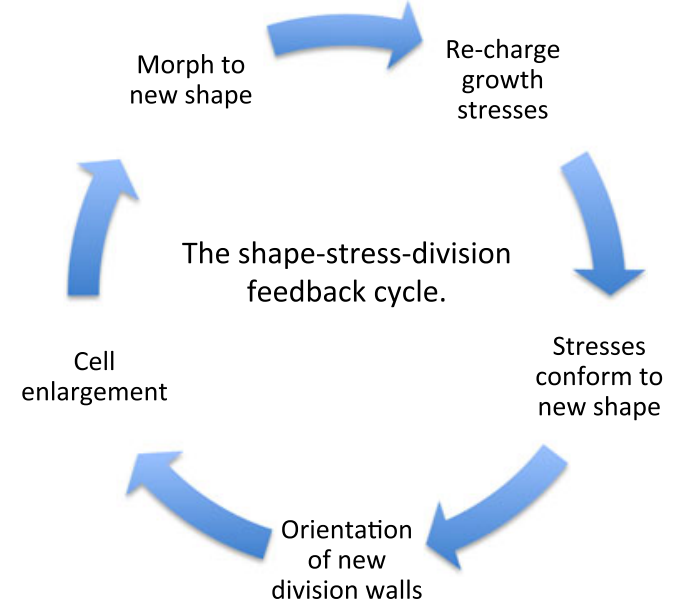

Fig. 2 Surface topology is a critical element in the cycle of shape changes that characterize apical morphogenesis. Cell division and growth leads to distortion of the apical surface, which redirects growth stress and reorients new partition walls

have been foreseen in earlier views of plant construction which invoked physical inputs in many aspects of cell shape and tissue patterning (Sinnott 1960). We are left with the conclusion that, because of the predictability of the relationship between shape and stress distribution and because of the ability of plant cells to interpret and respond precisely to physical inputs, we have the elements of a cyclic system of spontaneous and unscripted controls, an effective but nongenetic generator of repetitive shape changes.

The physical mechanics of the cell wall system has evolved over hundreds of millions of years. Because of this evolution, multicellular plants have gained access to a suite of highly tunable but spontaneous controls that have configured themselves such that they take control of mitotic division wall installations and directional cell enlargement in actively dividing tissues. Barring disturbance by some external influence, this epigenetic circuitry is self-sustaining and capable of governing the progressive transformation of one shape into another.

To summarize:

- In a turgid multicellular tissue, surface shape itself can determine the way growth stresses flow through the structure.

- The dividing cells respond by installing new partition walls in strict accordance with the trajectories of those stresses.

- Newly formed cells contribute their volumes to the emergence of new shape.

- The emergence of new shape remodels the distribution of growth stresses in the tissue, which reassigns the orientations of the next round of partition wall installations - and so on.

- Shape generates shape. 
These relationships, and the behaviors that they enable, are largely invisible to the casual observer and cannot be revealed with normal histological or biochemical analysis, but they are real nonetheless, and at least in principle, they are accessible to structural analysis by established engineering methods. By understanding that there is principal stress information implicit in the surface conformation of any growing plant organ, we can start to define a new perspective on the iteration of forms that characterize plant growth. This interpretation of meristematic ontogeny hints at an underlying structural logic of plant morphogenesis. But there are other aspects of plant development that may be equally revealing.

\section{Reproductive differentiation and the streptophyte sporangium}

In considering the significance of a biophysically driven developmental logic made available to plant evolution by virtue of the plant cell wall and the apoplastic continuity that it provides, there is one topic of particular significance that demands attention; that is, the nature of the inducing stimulus for reproductive differentiation. The lack of a suitable model for this most critical event in the life history of the streptophyte land plants constitutes a major gap in our understanding of plant reproduction. In particular, the mechanism or mechanisms underlying the ability of land plants to initiate the premeiotic differentiation of the archesporium remains one of the outstanding mysteries underlying plant sexual behavior and the alternation of generations.

To put this problem in perspective, we recall the distinction between sexual reproduction in plants and in animals. In animals, sexual differentiation revolves around the notion of a reserved germ line, which refers to the metazoan developmental trait of setting aside a number of progenitor cells very early in development that function as a source of gametes much later in the life cycle when the organism reaches reproductive maturity. The presumed advantage of this scheme is that, because these reserved progenitors divide infrequently or not at all while the surrounding somatic cells continue to multiply, they are not as likely to accumulate somatic mutations which might be disadvantageous were they to be transmitted to the next generation through the germ cells. Somatic mutations are thus minimized in the germ line simply by reducing the number of mitotic cycles that the germ line cells must endure (Grosberg and Strathmann 2007; Michod and Roze 2001).

But in the land plants, there is no germ line. Land plants develop clonally from a single cell-the zygote. There is no sequestration of germ cells. There are no cells in the embryo or in the young seedling that are predetermined to become germ cells or premeiotic sporocytes. Gamete formation begins with a singular differentiation event in a specialized sporangial structure that only appears at a relatively late stage in the life history of the sporophyte. Gametogenesis arises de novo out of a background of indistinguishable somatic cells. This leads to two obvious questions:

1. How do plants avoid accumulating deleterious somatic mutations, especially considering the extremely long life spans of the longest-lived plants?

2. How can gametogenesis be reliably initiated at the appropriate stage in the life history of the plant?

To answer the first question, we look to the meristematic growth habit characteristic of plants in which cell division becomes restricted to certain permanently embryonic regions, typically at the apical ends of the plant axis, where there may be one or more "initial cells" occupying a dominant position at the apical pole. Because these "initials" risk being swept out of the apex if they cannot keep up with their neighbors, they can be seen to be competing with each other for their position as progenitor cells (Klekowski 1988). In this way, deleterious mutations can be continually purged from the apical meristems. Secondly, because deviant cells are permanently imprisoned within their cell walls, they are unable to physically escape to another location where they might be able to avoid detection. And thirdly, because alternation of generations forces the life cycle of plants through a multicellular haploid generation which must compete for resources to a greater or lesser degree, there is an additional opportunity to purge nonconformists carrying maladaptive mutations (Walbot 1985; Walbot and Evans 2003).

The second question is more fundamental. Because plants develop clonally, with all somatic cells having continuously replicated their identical genomes by mitosis and with none having been set aside as a reserved germ line, there is no way to predetermine which cells will become the premeiotic archesporial cells. There is no cell carried forward into the flowering apex that is destined to differentiate into the archesporium. Premeiotic differentiation must, therefore, be initiated by some nongenetic singularity that tips one or a few equally competent and otherwise identical somatic cells towards a new developmental fate, leading to the initial differentiation of the archesporium and on through meiosis and gametogenesis (Lintilhac 1991).

Surprisingly, this most critical moment in the reproductive history of plants has never been definitively resolved, but its significance is central to the life histories of all land plants. It is the primary event that initiates all reproductive differentiation. Whatever the nature of the underlying mechanism, it must be able to reliably identify a single cell or a small group of cells precisely located within the young sporangium, successfully distinguishing them from their previously identical cohorts and neighbors, and to allow them to proceed to differentiate as the archesporium. All sexual reproduction in land plants can be traced back to this single event. Whether it 
is in the mosses or the orchids, the ferns, or the gymnosperms, the initiation of the sexual cycle poses the same developmental problem, namely, how does the plant create a precisely located and reliably initiated spatial singularity out of a background of genetic identity. The most commonly invoked hypothesis supposes that once competency has been set up in some local group of (sporangial) cells, induction is accomplished by some kind of diffusional gradient acting to pinpoint a cell or small group of cells (Kelliher and Walbot 2011)

But we tend to overlook the one feature that unites all reproductive differentiation in plants; that is, the architecture of the multicellular sporangium. The multicellular sporangium is found in all sexually competent streptophytes. It is a structure that can be traced back to the very first land plants (Gensel and Andrews 1984) where it appears in the fossil record even before we see evidence of true leaves. The sporangium combines all of the best spatial/mechanical attributes of the cell wall systems that we associate with higher plants in a single, actively growing, miniaturized structure. It boasts excellent cell-to-cell mechanical coupling, geometrical predictability (meaning repeatable and precise division patterns), and highly controlled surface shape, all packaged in a small symmetrical structure that is clearly capable of concentrating and focusing mechanical forces. It would seem to be well adapted to the task of reliably pinpointing small groups of somatic cells or even individual cells, in a mass of otherwise indistinguishable cohorts. Such a structure would seem to be ideally suited to setting up the spatial singularity that channels them towards their new fate as meiocytes (Figs. 3 and 4).

For the plant, the advantages of such a system are obvious because the signals are inherent in the geometry and growth characteristics of the structure. But for the experimentalist, the challenges are considerable. The structures are tiny at the

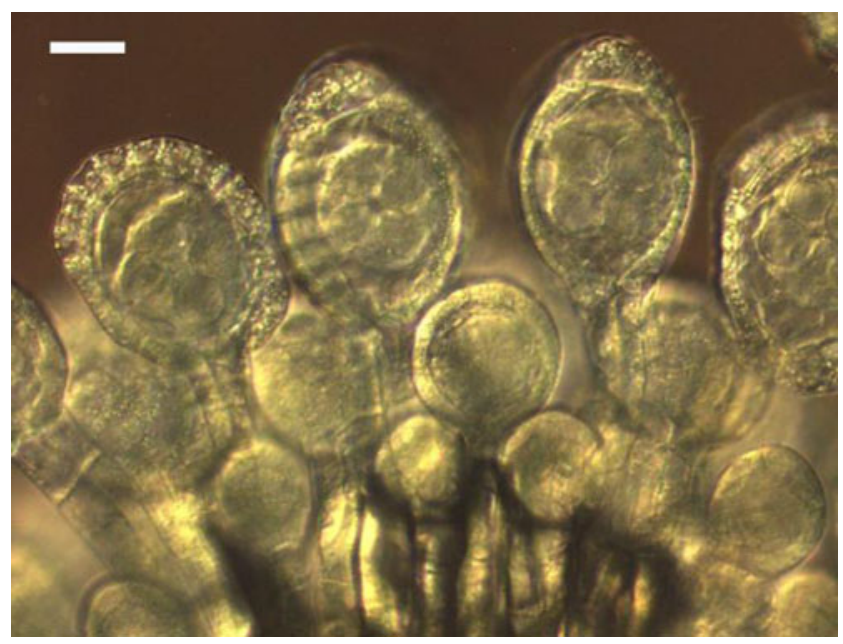

Fig. 3 Immature sporangia of the fern Cyrtomium sp. All land plant sporangia share common features. Note the distinct sporangial wall cells and central sporogenous region. Scale bar $=50 \mu$

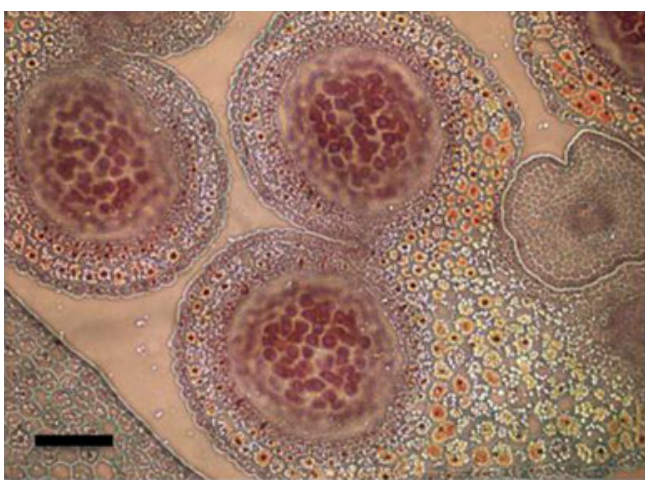

Fig. 4 Lilium sp. premeiotic anther sacs. Early anther development in the flowering plants shows striking similarities to sporangial development throughout the plant kingdom. Scale bar $=100 \mu$

time of archesporial differentiation, and there are no good tools and/or methods for dissecting the stress-mechanical relationships in such a small structure. However, once we admit the possibility that plant development arose through the biophysical evolution of the cell wall itself, then we can entertain the proposition that the streptophyte sporangium might be best understood as a stress-mechanical device that evolved specifically to resolve the germ line issue in plants. The multicellular sporangium, one of the truly defining features of all land plants, would seem to be ideally configured to redirect growth stresses, effectively serving as a simple stress-mechanical lens focusing mechanical signals on a single cell or a small group of cells, independent of temperature, hormonal gradients, or other diffusible chemical morphogens. ${ }^{9}$ Again, we are presented with the notion that, in the plant kingdom, the evolution of multicellularity and reproductive differentiation are manifestations of the evolution of the cell wall itself. Evolution has co-opted the biophysics of enclosed pressure vessels and complex nanostructural composites. In this way, the basic transformations of morphogenesis can be maintained using simple but sophisticated control cycles without the need to continually retrieve archived step-by-step instructions from the genome in order to proceed from one configuration to the next.

\section{Conclusion}

The development of form in plants can be regarded as the manifestation of a kind of reciprocating morphogenetic engine. An engine whose trajectory through time engages a series of biophysically based logic circuits that are sufficiently stable

\footnotetext{
${ }^{9}$ The proposition that the multicellular sporangium acts to trigger idiosyncratic differentiation of the germ cells in plants by biophysical means has been raised several times by this author (Lintilhac 1974, 1984), but the extremely small size of living pre-archesporial sporangia makes experimental confirmation difficult.
} 
and self-correcting to channel successive generations along similar ontogenesis, resulting in a repeatable sequence of developmental forms. Supporting this engine are a variety of other embedded modular control processes, some of them also cyclic, which are active at various lower hierarchical levels. We have given these other modules names such as oxidative phosphorylation, photosynthesis, membrane transport, cytoskeleton, and the plethora of molecular mechanisms responsible for the transcription and translation of genetic material. ${ }^{10}$

So, what is the role of the genome itself, and how do we understand the governing roles of the many cellular and subcellular control circuits that are embedded in the living system that we call the organism? When it comes to interpreting the flow of morphological forms that constitute development, do we look for scripted instructions in the genome when the most relevant control circuitry seems to be inherent in the architecture of the developing organ and the biophysical properties of the cell wall itself?

Looking for a master controller which integrates all these subsystems under one set of scripted instructions, we find that we are drawn to one or another of the basic modules of cellular function because of its ability to control energy flow and maintain correct timing or because mutational analysis shows us that it alters the phenomenology of development in some way. But in fact, there may be no master controller. It may be more fruitful to regard morphogenesis as a continuously selfregulating flow of spontaneous transitions, with each step in the development of the mature organism depending solely on the configuration of the system in the previous moment. We may interpret one or another of the lower-order subsystems as being more or less scripted and, therefore, reminiscent of a choreographer acting behind the scenes to ensure the continuity of the performance; but in reality, there may be no choreographer. There is only time, repetition, and evolutionary survival.

Lastly, in the study of plant development, we may have a opportunity to articulate a comprehensive narrative that frames developmental ontogeny and evolution in a way that is not currently possible in the animal kingdom where multiple levels of complexity obscure the logic of developmental ontogeny. In plants, the logic of development is constrained by the universality of the cell wall system. The restriction on cell movement imposed by the apoplast is offset by the possibility that it has enabled the evolution of a precise set of mechanical behaviors, operating independently of genetic developmental programming, and which are directly responsible for the life histories that we see in land plants. Because of these same constraints, we may be able to envision a

\footnotetext{
${ }^{10}$ Niklas and Kutschera (2012) approach this question in terms of the Subsystem Incompleteness Theorem which says that "no subsystem is operationally self-sufficient." Which is to say: no subsystem can predict or control development at the organismal level.
}

general paradigm for morphogenesis that integrates the cellular and molecular aspects of plant biology under a comprehensive structural logic that has driven the evolution of multicellular form.

The three major components of plant developmental ontogeny, namely, axial growth, apical morphogenesis, and reproductive differentiation, can be framed in the context of spontaneous biophysical control circuits that are deterministic but unscripted, leaving us with the conjecture that the transitions between the successive forms that constitute plant morphogenesis are in reality completely spontaneous and not programmed in any meaningful sense at the level of the genome or anywhere else.

Conflict of interest The author declares that he has no conflict of interest.

Open Access This article is distributed under the terms of the Creative Commons Attribution License which permits any use, distribution, and reproduction in any medium, provided the original author(s) and the source are credited.

\section{References}

Brown CL (1964) The influence of external pressure on the differentiation of cells and tissues cultured in vitro. In: Zimmermann MH (ed) The formation of wood in forest trees. Academic Press, New York

Dorrington K (1980) The theory of viscoelasticity in biomaterials. In: Vincent JFV, Currey JD (eds) The mechanical properties of biological materials. 34th Symposium of the Society of Experimental Biology

Eigen M, Schuster P (1979) The hypercycle: a principle of natural selforganization. Springer, Berlin

Errera L (1888) Über Zellformen und Ziefenblasen. Bot Centralbl 34:395-398

Gensel P, Andrews H (1984) Plant life in the Devonian. Praeger, New York Graham L (1993) Origin of land plants. Wiley, New York

Grosberg R, Strathmann R (2007) The evolution of multicellularity: a minor major transition? Ann Rev Evol Syst 38:621-654

Hamant O, Heisler G, Jonsson H, Kurpinski PK, Uyttewaal M, Bokov P, Corson F, Sahlin P, Boudaoud A, Meyerowitz E, Couder Y, Traas J (2008) Developmental patterning by mechanical signals in Arabidopsis. Science 322:1650-1655

Hejnowicz Z (1984) Trajectories of principal directions of growth, natural coordinate system in a growing plant organ. Acta Soc Bot Pol 29-42

Hejnowicz Z, Karczewski J (1993) Modeling of meristematic growth of root apices in a natural coordinate system. Am J Bot 309-315

Hepler P (1971) The role of microtubules in vessel member differentiation in Coleus. Protoplasma 72:213-236

Heywood RB (1969) Photoelasticity for designers. Pergamon, Oxford

Hofmeister W (1863) Zustäze und Berichtigungen zu den 1851 veröffentlichen Untersuchungen der Entwicklun höherer Kryptogamen. Jahrb Wiss Bot 3:259-293

Katchalsky A, Curran P (1965) Nonequlibrium thermodynamics in biophysics. Harvard University Press, Cambridge

Kaplan D, Cooke TJ (1996) The genius of Wilhelm Hofmeister: the origin of causal-analytical research in plant development. Am J Bot 83:1647-1660 
Kaplan D, Hagemann W (1991) The relationship of cell and organism in vascular plants: are cells the building blocks of plant form? Bioscience 41:693-703

Kelliher T, Walbot V (2011) Emergence and patterning of the five cell types of the Zea mays anther locule. Dev Biol 350:32-49

Klekowski E (1988) Mutation, developmental selection, and plant evolution. Columbia University Press, New York

Lintilhac P (1974) Differentiation, organogenesis, and the tectonics of cell wall orientation. III. Theoretical considerations of cell wall mechanics. Am J Bot 61:230-237

Lintilhac P (1984a) Positional controls in meristem development: a caveat and an alternative. In: Barlow PW, Carr DJ (eds) Positional controls in plant development. Cambridge University Press, Cambridge

Lintilhac P, Vesecky T (1981) Mechanical stress and cell wall orientation in plants. II. The application of controlled directional stress to growing plants: with a discussion on the nature of the wound reaction. Am J Bot 68:1222-1230

Lintilhac P (1991) Towards a theory of cellularity: speculations on the nature of the living cell. Bioscience 49:59-67

Lintilhac P (1984b) Stress induced alignment of plant tissues grown in vitro. Nature 307:363-364

Lynch T, Lintilhac P (1997) Mechanical signals in plant development: a new method for single cell studies. Devel Biol 181:246-256

Lyndon RF (1994) Control of organogenesis at the shoot apex. New Phytol 128:1-18

Magallon S, Hilu KW, Quandt D (2013) Land plant evolutionary timeline: gene effects are secondary to fossil constraints in relaxed clock estimation of age and substitution rates. Am J Bot 100:556-573

Metraux J, Taiz L (1978) Transverse viscoelastic extension in Nitella. 1. Relationship to growth rate. Plant Physiol 61:135-138

Michod RE, Roze D (2001) Cooperation and conflict in the evolution of multicellularity. Heredity $86: 1-7$

Mueller B (1952) Goethe's botanical writings. University of Hawaii Press, Honolulu

Nakashima K, Yamada L, Satou Y, Azuma J, Satoh N (2004) The evolutionary origin of animal cellulose synthase. Dev Genes Evol 214:81-88

Nick P (2011) Mechanics of the cytoskeleton. In: Wojaszedk P (ed) Mechanical integration of plant cells and plants. Springer, Berlin, pp 53-90

Nick P (2000) Control of plant shape. Plant microtubules: potential for biotechnology. Springer, Heidelberg
Niklas K (2004) The cell walls that bind the tree of life. BioScience 54:831-841

Niklas KJ, Kutschera U (2012) Plant development, auxin, and the subsystem incompleteness theorem. Frontiers in Plant Sci 3:1-11

Panovko YG, Gubanova II (1965) Stability and oscillations of elastic systems. Consultants Bureau, New York

Plateau JAF (1873) Statique expérimentale et théorique des liquides soumis aux seules forces moléculaires. Gauthier-Villars, Paris (2 volumes)

Rzhanitsyn AR (1955) Stability of the equilibrium of elastic systems. Gostekhizdat, Moscow

von Sachs J (1878) Über die Anordnung der Zellen in jüngsten Pflanzentheilen. Arb Bot Inst Würzburg 2:46-104

Scheiner SM (2010) Toward a conceptual framework for biology. Q Rev Biol 85:293-318

Schrödinger E (1944) What is life? Cambridge University Press, Cambridge

Selker J, Steucek G, Green P (1992) Biophysical mechanisms for morphogenetic progressions at the shoot apex. Dev Biol 153:2943

Sinnott EW (1960) Plant morphogenesis. McGraw-Hill, New York

Szymanowska-Pulka J, Potocka I, Karczewski J, Jiang K, Nakielski J, Feldman L (2012) Principal growth directions in development of the lateral root in Arabidopsis thaliana. Annals of Botany (Special Issue: Root Biology) 110:1-11

Taiz L (1984) Plant cell expansion: regulation of cell wall mechanical properties. Ann Rev Plant Physiol 35:585-657

Takhtajan A (1991) Evolutionary trends in flowering plants. Columbia University Press, New York

Tomos A, Leigh R (1999) The pressure probe: a versatile tool in plant cell physiology. Annu Rev Plant Physiol Plant Mol Biol 50:447-472

Walbot G (1985) On the life strategies of plants and animals. Trends Genet 1:165-169

Walbot V, Evans M (2003) Unique features of the plant life cycle and their consequences. Nature Reviews Gen 4:369-379

Wei C, Lintilhac P (2003) Loss of stability - a new model for stress relaxation in plant cell walls. J Theor Biol 224:305-312

Wei C, Lintilhac P (2007) Loss of stability: a new look at the physics of cell wall behavior during plant cell growth. Plant Physiol 145:763-772

Wojtaszek P (2001) Organismal view of a plant and a plant cell. Acta Biochim Polonica 48:443-451 